\title{
DOS CUPAS COMPLUTENSES
}

\author{
POR \\ ARMIN U. STYLOW \\ Centro CIL II, Universidad de Alcalá
}

\section{RESUMEN}

Se publican dos cupas del antiguo territorio de Complutum, una, inédita, encontrada en excavación en Alcalá de Henares (Madrid), y la segunda, procedente de la iglesia de Alovera (Guadalajara), con una nueva lectura.

\section{ABSTRACT}

Publication of two cupae from the ancient territory of Complutum. One was found in excavations in Alcalá de Henares (Madrid) and is unpublished; of the other one, which came to light in the church of Alovera (Guadalajara), a new reading is given.

PALABRAS CLAVE: Complutum, cupa, epigrafía latina.

KEY WORDS: Complutum, cupa, Latin epigraphy.

\section{CUPA DE ALCALÁ DE HENARES ${ }^{1}$}

En el curso de las excavaciones realizadas en un solar situado inmediatamente al sur de la actual Puerta de Madrid de Alcalá de Henares, se encontró, en la primavera del año 2001, una cupa funeraria. Si bien ese solar está enclavado en la zona de la necrópolis oriental de la ciudad romana de Complutum, que se extendía a ambos lados de la calzada Complutum-Caesaraugusta (cf. Rascón Marqués, 1995, 150 fig. 57) la cupa misma apareció fuera de contexto arqueológico, en superficie y mezclada con materiales de construcción moderna; concretamente había sido reutilizada como basa de uno de los varios pilares que sustentaban el techo de unas cuadras construidas en el solar. Aun así, es sumamente probable que proceda en último lugar de dicha necrópolis ${ }^{2}$. La pieza fue trasladada al depósito

\footnotetext{
1 Trabajo realizado en el marco de los proyectos 06/0152/2000 de la Comunidad de Madrid y BFF2003-04778 de la DGCyT.

2 Agradecemos a César Manuel Heras Martínez, director de la excavación, sus informaciones sobre las circunstancias del hallazgo y a Sebastián Rascón Marqués, Director del Servicio de Arqueología del Ayuntamiento de Alcalá de Henares, las facilidades dadas para su estudio. A ambos van nuestros agradecimientos por el permiso de publicar la pieza.
}

del T. E. A. R. de Alcalá de Henares, donde se conserva en la actualidad y donde la pudimos estudiar en mayo del 2001 y otra vez en octubre de este año, cuando realizamos la fotografía que presentamos aquí (fig. 1).

La cupa, labrada en piedra caliza dura gris de origen local, presenta la habitual forma paralelepipédica con la parte superior redondeada. Mide $46 \mathrm{~cm}$ de alto, $42 \mathrm{~cm}$ de ancho y $72 \mathrm{~cm}$ de grueso. Toda la superficie está someramente alisada. La cara frontal, la que lleva la inscripción, está rematada en ambos lados por sendas acróteras, rasgo que se repite en otras cupas complutenses ${ }^{3}$; la acrótera derecha se conserva sólo en parte, debido a la fractura que ha afectado a la parte derecha frontal de la cupa y que también ha dañado los finales de las 1. 2-5. En el centro de los laterales presenta la cupa sendos hoyos que habrían servido para encajar en ellos las pinzas de la grúa que sería necesaria para mover una pieza de semejante tamaño y peso. En época posterior se practicaron dos agujeros de sección elíptica en la parte alta de la cara frontal, cuya finalidad desconocemos; uno de ellos ha destruido la mitad superior de la letra D de 1. 1. Esos agujeros están parcialmente rellenos de mortero, y más adherencias de mortero se encuentran en varias partes de la cupa.

La incisión de las letras de la inscripción es poco regular y, en general, bastante tosca, variando su altura desde los 5,5 cm de las 1. 1, 4 y 5 (en esta última línea, sin embargo, no es posible averiguar la altura original exacta, puesto que su extremo inferior ha sufrido un fuerte desgaste), hasta los $7 / 10 \mathrm{~cm}$ de las 1.2 y 3 . Parece existir una sola interpunción, triangular, entre las dos letras de la 1. 1, aunque también podría tratarse de una cavidad natural de la piedra. Hay un nexo de A y $\mathrm{N}$ al principio de la 1. 4. El texto reza:

\author{
$D($ is $) \cdot M($ anibus $)$ \\ Ter $<e>n t i(a) e$ \\ Antil( a)e \\ ân(n)orum) $[-4 ?-]$ \\ $5 s(i t) t($ ibi) (vac. 3) t(erra) [l(evis)]
}

\footnotetext{
3 Knapp, 1992, 127 (= CIL II 3036). 164. Más frecuentes, sin embargo, son esas acróteras en las estelas complutenses, desde donde su utilización puede haber pasado a las cupas.
} 


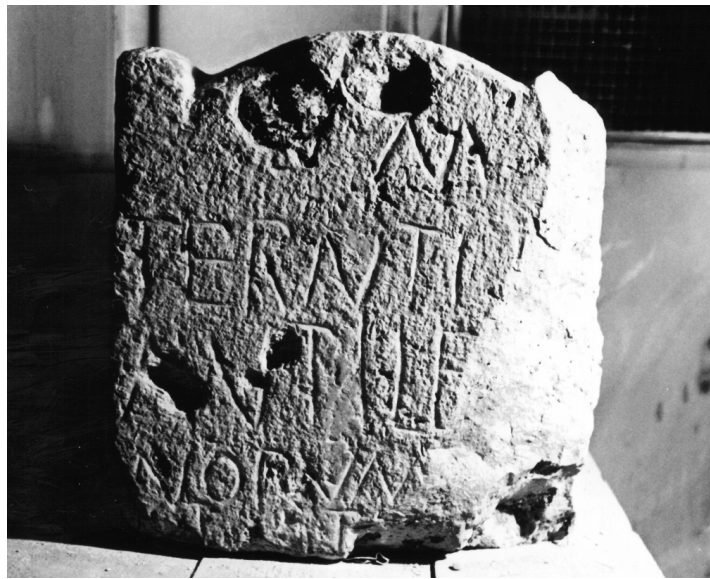

Figura 1. Cupa de Alcalá de Henares (fotografía del autor).

En la 1. 2, es evidente que, al grabar el gentilicio, han omitido la letra $\mathrm{E}$ entre $\mathrm{R}$ y $\mathrm{N}$; es posible que hubieran tenido la intención de escribirla con un nexo, bien de RE o bien de EN, pero el caso es que ese nexo tampoco se realizó. El gentilicio Terentius, aunque bien representado en toda Hispania (Abascal Palazón, 1994: 227 ss.), es -por ahora- nuevo en la epigrafía complutense. Al final del renglón, en la zona perdida por la fractura, quedaría espacio para restituir unas dos letras más, que entonces tendrían que corresponder o a una filiación o, si de una liberta se tratara, a la indicación del patrono; sin embargo, ninguna de esas alternativas es verosímil, no sólo por la cronología de la inscripción (cf. abajo), que ya no deja esperar semejantes indicaciones, sino también porque, si nuestra restitución es acertada, un espacio parecido -e incluso mayor, de unas 3-4 letras- habría existido después de la última letra conservada al final de la 1. 3, para el cual sería difícil proponer una restitución satisfactoria. Más bien parece que el cuerpo principal de la inscripción -a excepción de la 1 . 1, centrada- estaba alineado rigurosamente por la izquierda, dejándose sin inscribir los huecos resultantes al final de los renglones. Esa reconstrucción encontraría un apoyo en los mayores espacios que se han dejado entre la I y la $\mathrm{E}$ al final de la 1. 2 (una semejante tendencia a espaciar las letras se observa también hacia el final de la 1. 3) y, sobre todo, entre las dos T de la última línea, con los que se habría pretendido compensar en parte ese desequilibrio de la ordinatio. El cognomen Antila sólo aparece una vez más en todo el Imperio Romano, y precisamente en una inscripción de Complutum ${ }^{4}$; si no es un nombre indígena, para el que faltarían paralelos, parece tratarse más bien

\footnotetext{
4 AE 1985, 606; para la interpretación del texto cf. ahora Gómez-Pantoja, 2001, 257-263. Abascal Palazón, 1994, no recoge este testimonio.
}

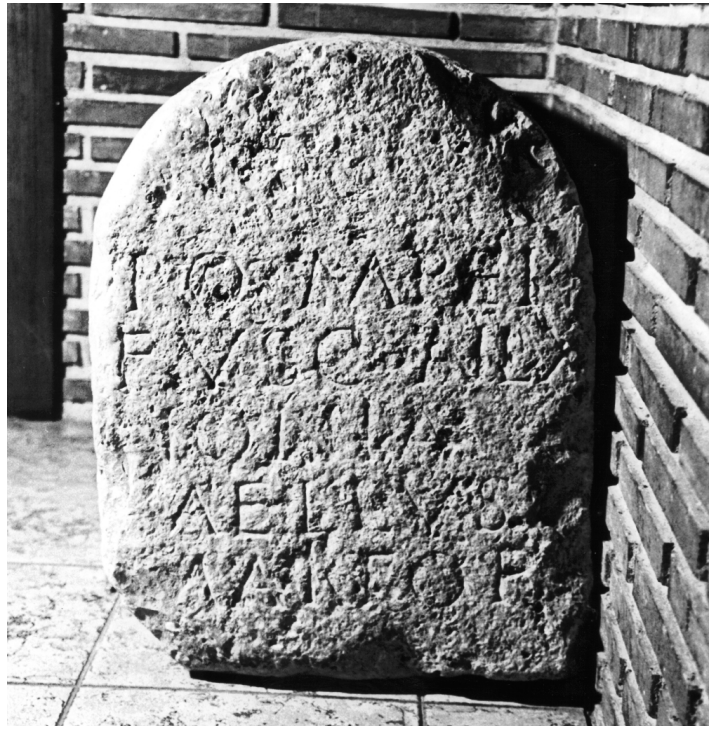

Figura 2. Cupa de Alovera (fotografía del autor).

de una variante de Antulla ${ }^{5}$ que de una grafía vulgar del nombre griego Anthylla.

Tanto la paleografía como la onomástica y las fórmulas utilizadas (p. ej., falta de h. s. e.) apuntan hacia una datación en la primera mitad del siglo III.

\section{CUPA DE ALOVERA}

Hace poco publicó Abascal Palazón, J. M. (1999, 297 s.) la inscripción de una cupa que se encontró en el año 1999 al hacerse obras dentro de la torre de la iglesia de Alovera (Guadalajara), población situada a unos 15 km en línea recta al noreste de Alcalá de Henares y cuyo término, a todas luces, formaba parte del territorio antiguo de la ciudad de Complutum (fig. 2). Abascal da la siguiente transcripción del texto:

Pompei
Fusci an(norum) LX
Antonia
Maellusa
marito $p$ (osuit)

Habría nexos de AN en la 1. 2 (para la cual Abascal además anota una "O parva", que no se refleja en su transcripción), de ANT en la 1. 3, y de MA en las 1. 4 y 5.

5 En este sentido se expresa también Knapp, 1992, 148; para Antullus cf. Kajanto, 1965, 175. Gómez-Pantoja plantea como explicación alternativa que sea un derivado del gentilicio Antilius, lo que parece menos probable. 
En el mes de julio de 2001, nosotros tuvimos ocasión de estudiar la cupa, ahora conservada en el Ayuntamiento de Alovera ${ }^{6}$, en óptimas condiciones, lo que nos permite ampliar ahora su descripción. El material en el que se talló es una caliza dura local, medio metamorfizada y por lo tanto pulimentable (así que, para los romanos, sería marmor). Además de los desperfectos que presenta en los bordes laterales y en la esquina inferior izquierda, con la consiguiente pérdida parcial de algunas letras extremas de la inscripción, está rota abajo en la parte posterior, someramente desbastada, y tiene un hoyo cónico no muy profundo -antiguo, por lo que parece- en la parte central de su dorso. Ese hoyo -más que un agujero para fijar mejor las pinzas de una grua con que mover la piedra, como los que conocemos en otras cupas de Complutum, donde, sin embargo, normalmente se encuentran en los laterales (cf. la nueva cupa descrita arriba)- puede haber servido para encajar en él una estructura metálica como, p. ej., un focus de bronce para los sacrificios en honor y conmemoracición del difunto, costumbre bien atestiguada por varias cupas de Augusta Emerita.

Abascal vio la pieza, al poco tiempo de desenterrarla, al aire libre, sin la luz rasante artificial de la que disponíamos nosotros, y esto explica seguramente que, por un lado, haya interpretado como trazos grabados algunos desconchones y otros desperfectos de la piedra causados por la erosión y que, por otro, haya pasado por alto algunas letras grabadas a menor tamaño. El que el nombre del difunto se expresara, según su lectura, en genitivo, caso que dependería de un sustantivo sobrentendido como monumentum, sería extraordinario en Complutum, donde en toda la epigrafía funeraria no aparece un genitivo más que una vez, dependiendo de la fórmula Dis Manibus (CIL II 3034 = Knapp, 1992, 139). Por el contrario, el nominativo es el caso normal para nombrar al difunto, excepción hecha, naturalmente, de los ejemplos donde el nombre está en dativo, bien como objeto indirecto de la acción -implícita o expresa- realizada por el responsable del entierro y de la erección del monumento, o bien -en las inscripciones tardías, como la de la nueva cupa de Alcalá de Henares que damos a conocer en la primera parte- sin la mención del dedicante. Es al primero de esos esquemas al que corresponde también la inscripción de la cupa de Alovera, cuya cronología está comprendida entre finales del siglo I y mediados del II. Su texto reza (fig. 2; foto: Stylow):

\footnotetext{
${ }^{6}$ Agradecemos a la alcaldesa de Alovera, D ${ }^{\mathbf{a}}$. M ${ }^{\mathrm{a}}$. del Carmen Plaza Castro, la autorización y las facilidades que nos ha dado para el estudio de la pieza.
}

\author{
Pompeio \\ Fusco $\cdot \hat{a n}($ norum $) \cdot L X$ \\ Nonia \\ Mellusa \\ 5 marito $f$ (aciendum) [c(uravit)]
}

Tanto el gentilicio como el cognomen del difunto terminan con una $\mathrm{O}$ pequeña que sólo tiene $2,5 \mathrm{~cm}$ de alto, la mitad de altura de las demás letras. En la segunda línea se encuentran los únicos signos de interpunción del texto, pequeños triángulos, de los que sólo el primero es seguro. Cabe pensar que se pusiesen porque la línea contiene más de una palabra y quisieran evitar confusiones; en la 1. 5, sin embargo, no hay interpunción.

Los supuestos nexos de letras al principio de las líneas 3-5 no existen. La forma de la primera $\mathrm{N}$ de Nonia (1. 3) es prácticamente idéntica a la de la segunda y no presenta la ligera inclinación hacia la derecha que se percibe en el nexo AN de la 1. 2; los travesaños de A y $\mathrm{T}$ que Abascal quería ver en esa $\mathrm{N}$ no son más que desconchones de la piedra, no trazos de letras incisos a bisel. Lo mismo vale para el supuesto nexo MA en la l. 4. La A final se ha perdido casi enteramente y no queda más que un mínimo resto del arranque del primer trazo. $\mathrm{Al}$ principio de la 1. 5, finalmente, lo que se conserva son los tres últimos trazos de una M situada exactamente debajo de la $\mathrm{M}$ de la 1. 4, y luego, claramente separada por un espacio, una A; aquí, por lo tanto, tampoco hubo ningún nexo.

Por otro lado, conviene destacar el único nexo verdadero de la inscripción, de las letras RI en la 1. 5, muy elegante (las letras en general están muy bien grabadas, siendo deficiente por otro lado su distribución armoniosa en las líneas), con el bucle de la R que no toca la I longa. La última letra de esa línea no es una $\mathrm{P}$, como dice Abascal y como puede aparecer en la foto, sino una $\mathrm{F}$, cuyos dos travesaños, igual que en la F de 1. 2, muestran unos refuerzos finales muy marcados que casi se juntan y pueden producir esa impresión equivocada. Un $p$ (osuit) a secas sería, además, totalmente singular en la epigrafía funeraria complutense ${ }^{7}$, donde la fórmula estándar con la que se designa la actividad de los dedicantes de los monumentos es $f$ (aciendum) c(uravit) ${ }^{8}$; por lo cual no dudamos en restituir esa fórmula también aquí. La C final se habrá perdido en la rotura del extremo derecho de la cupa.

Con la nueva lectura, la onomástica de los personajes nombrados encaja perfectamente en el ambiente complutense. Mientras que hasta ahora no se conocen allí unos Antonii, los Nonii estaban ya atestiguados por

7 El poni volo de la inscripción Knapp, 1992, 148 tiene otro contexto, cf. abajo, nota 11 .

${ }^{8}$ Cf. Knapp, 1992, 126 ss. 
dos inscripciones, siendo una de ellas, curiosamente, la única en que aparezca un magistrado complutense ${ }^{9}$. Si bien la Pompeia Mellusa de la cupa de Alovera, por su cognomen griego, más bien parece ser una liberta, conocemos a algunos Pompeii complutenses que claramente pertenecían a la élite local. Incluso es así que el único pedestal de estatua de la ciudad que se ha conservado ${ }^{10}$ es de una Pompeia Antila, dedicado tras su muerte por su hijo ${ }^{11}$.

En cuanto a la forma del monumento, no es tan infrecuente en esta zona de la Meseta como afirma el editor: con la nueva pieza de Alcalá de Henares ya son nueve las cupas que se conocen de Complutum y su territorio $^{12}$. Su forma sencilla algunas veces es enriquecida por unas acróteras laterales en la cara frontal ${ }^{13}$. Los ejemplos complutenses se integran pues en el centro de esa amplia banda formada por la zona de distribución de las cupas, que atraviesa la Península en diagonal desde el Alentejo portugués y la Andalucía occidental y, pasando por Extremadura, Ávila y los valles de los ríos Henares y Jalón, llega hasta Cataluña, con algunos ejemplares aislados fuera de esa banda, como los de Astorga, Lugo y Cartagena ${ }^{14}$.

9 CIL II 3033; la otra es CIL II 3042. Un Nonius es posiblemente también el $N(-$ - -) $C(-$ - ), dueño del esclavo Lucifer, que aparece en una inscripción inédita de Alcalá de Henares (cf. la fotografía y la traducción castellana del texto que di a conocer en el Diario de Alcalá del 5 de febrero de 2004).

${ }^{10}$ Otro fue probablemente el del mencionado magistrado complutense, que se ha perdido.

${ }^{11}$ Knapp, 1992, 148. Para su interpretación correcta véase Gómez-Pantoja, 2001.

${ }^{12}$ Además de esas dos conocemos tres de la misma Alcalá de Henares (Knapp, 1992, 127, 135 y 137), una de San Fernando de Henares (Knapp, 1992, 164), una de Guadalajara (Abascal y Cebrián, 2004/05, 199-200) y dos de Alcolea de Torote (AE 1987, 632 y 633). Pueden haber existido más ejemplares, que no se hayan identificado como tales, puesto que se han perdido y su forma no queda reflejada en las descripciones antiguas.

${ }^{13}$ Como, p. ej., en la nueva cupa de Alcalá de Henares, cf. arriba.

${ }^{14}$ Véase la amplia documentación en Abascal, 1995, 75 s.; Abascal y Ramallo, 1997, 33 s.; cf. Stylow, 2002, 177. Ruiz Trapero $(2001,31)$ atribuye la distribución de las cupas a las comunicaciones a través de la red viaria romana y piensa incluso en el trabajo de lapidarii procedentes de otros entornos geográficos, lo que parece poco probable en vista de que cada zona desarrollaba subtipos propios muy característicos. Un estudio de la totalidad del material peninsular está en preparación por parte de Charlotte Tupman de la Universidad de Southampton, bajo la dirección de Simon Keay.

\section{BIBLIOGRAFÍA}

ABASCAL PALAZÓN, J. M., 1994: Los nombres personales en las inscripciones latinas de Hispania. Murcia.

ABASCAL, J. M., 1995: Las inscripciones latinas de Santa Lucía del Trampal (Alcuéscar, Cáceres) y el culto de Ataecina en Hispania. AEspA, 68, 31-105.

ABASCAL, J. M., 1999: Apuntes epigráficos (Mirobriga, Ilici, Segobriga, Saldeana, Carpio de Tajo y Alovera). AEspA, 72, 287-298.

ABASCAL PALAZÓN, J. M. y CEBRIÁN FERNÁNDEZ, R., 2004/05: Inscripciones de Guadalajara, Ávila, Alconétar, Segobriga y Sevilla en manuscritos de la Real Academia de la Historia. Lucentum, 23/24, 197-205.

ABASCAL, J. M. y RAMALLO, S. F., 1997: La ciudad de Carthago Nova III. La documentación epigráfica. Murcia.

GÓMEZ-PANTOJA, J., 2001: Un olvidado fideicomiso de Complutum, Ocio y espectáculo en la Antigüedad Tardía. Actas del II Encuentro "Hispania en la Antigüedad Tardía” - Alcalá de Henares, 15 a 17 de Octubre de 1997 (Acta Antiqua Complutensia II). Eds. García Moreno, L. y Rascón Marqués, S. Alcalá de Henares, 257-263.

KAJANTO, I., 1965: The Latin cognomina. Helsinki.

KNAPP, R. C., 1992: Latin inscriptions from Central Spain. Berkeley - Los Angeles - Oxford.

RASCÓN MARQUÉS, S., 1995: La ciudad hispanorromana de Complutum. Alcalá de Henares.

RUIZ TRAPERO, M ${ }^{\mathrm{a}}$., 2001: Inscripciones Latinas de la Comunidad Autónoma de Madrid (siglos I-VIII). Madrid.

STYLOW, A. U., 2002: Von der Schrift der Sieger zum Sieg der Schrift. Imitation, Eigenständigkeit und Differenzierung in der epigraphischen Kultur Hispaniens. Convegno Hispania omnibus terris felicior. Premesse ed exiti di un processo di integrazione. Atti del convegno internazionale, Cividale del Friuli, 27-29 settembre 2001. Ed. G. Urso. Pisa, 163-181.

Recibido el 06-06-06 Aceptado el 10-09-06 